\title{
PSYCHOPHARMACOLOGICAL SIGNATURES IN THE RETINA IN SCHIZOPHRENIA AND BIPOLAR DISORDER: AN OPTIC COHERENCE TOMOGRAPHY STUDY
}

\author{
Ilkay Keles Altun ${ }^{1}$, Neslihan Turedi ${ }^{2}$, Neriman $\operatorname{Aras}^{3} \&$ Murat Ilhan Atagun ${ }^{4}$ \\ ${ }^{I}$ Department of Psychiatry, Bursa Yüksek Ihtisas Research and Training Hospital, Bursa, Turkey \\ ${ }^{2}$ Department of Ophtalmology, Trabzon Kanuni Research and Training Hospital, Trabzon,Turkey \\ ${ }^{3}$ Department of Psychiatry, Samsun Psychiatric, Samsun, Turkey \\ ${ }^{4}$ Department of Psychiatry, Izmir Bakircay University Faculty of Medicine, Izmir, Turkey
}

received: 26.10.2020; $\quad$ revised: 28.11.2020; $\quad$ accepted: 2.12 .2020

\begin{abstract}
SUMMARY
Background: Retina is considered as a window to the brain due to the similarities in terms of development and pathologies. Optical coherence tomography (OCT) can perform quantitative examinations in the retina. In this study, we aimed to investigate the effects of drugs used in schizophrenia and bipolar disorder (BD) on retinal nerve fiber layer (RNFL) and macular thickness.

Subjects and methods: The study included schizophrenia $(n=35)$ and euthymic $B D(n=46)$ patients on various medications, and age, gender matched healthy control group $(n=31)$. For retinal evaluation, measurements of RNFL and macula were performed with Optovue RTVue Premier OCT.

Results: In the schizophrenia group, chlorpromazine equivalent dose of antipsychotics was a statistically significant negative predictor of left RNFL nasal superior region thickness. In the BD group, serum valproate level was a significant positive predictor of thickness in the right macular inferior outer, left macular nasal outer region, right RNFL inferotemporal, left temporal and inferotemporal regions.

Conclusion: Since the retina consists of neurons, morphological or functional examination of retina may be beneficial for the evaluation of the effects of psychopharmalogical treatments in schizophrenia and BD. The outcome of this study implies that valproate has neuroprotective effects on the optic nerve and macula, and this finding is consistent with the literature implying neurotrophic effects of valproate.
\end{abstract}

Key words: schizophrenia - bipolar disorder - retina - valproate - antipsychotics

$* * * * *$

\section{INTRODUCTION}

Bipolar disorder (BD) and schizophrenia are mental disorders that cause severe dysfunction (Whiteford et al. 2015). Although there are differences in the clinical course between the two disorders, similarities can be observed in clinical and neurobiological variables. Some common neurobiological changes have been seen in structural neuroimaging studies and postmortem studies, decrease in brain volume, loss of myelin in white matter tracts, voltage loss in electroencephalography experiments, and neuronal loss in postmortem histopathological examinations (Gigante et al. 2011, Honea et al. 2005, Kempton et al. 2008, Narayanan et al. 2013, Nudmamud et al. 2003).

According to the neurodegenerative hypothesis, disruptions in the vital functions of cells result in volume loss in the tissues at the end of a process that can be defined as premature aging (Buoli et al. 2017). The severity of symptoms may be related to the severity of neurodegenerative changes (Moorhead et al. 2007). Since it is a neural tissue, the retina also has the potential to be affected by such changes and many findings supporting this have been obtained in recent years (Thiele et al. 2018).
Optic coherence tomography (OCT) is a non-invasive device used in retinal examination in clinical practice. It can reveal retinal layers with very good consistency and precision. One of these measures, Retinal Neural Fiber Layer (RNFL) has been used for evaluations not only in ophthalmological disorders but also in various diseases that progress with neurodegeneration such as multiple sclerosis, Parkinson's and Alzheimer's disease (Lu et al. 2010, Moschos et al. 2011). There are studies reporting different results regarding RNFL and macular thickness in schizophrenia and BD. Some studies have reported that the RNFL is thinned in schizophrenia and BD (Ascaso et al. 2015, Martin et al. 2019, Pan et al. 2018), and others have found no difference (Chu et al. 2012, Topçu-Yilmaz et al. 2019). In a meta-analysis of 20 studies, the eyes of 541 schizophrenia patients, 279 BD patients and 904 healthy control subjects were examined, and it was reported that the RNFL was observed to be thinner in the schizophrenia and BD groups compared to the healthy control groups (Liziano et al. 2020). Furthermore, retinal microvascular abnormalities have also been observed in schizophrenia and BD (Appaji et al. 2019, Yusifova et al. 2020).

Since it contains neural cells, neurotransmitters such as acetyl choline, dopamine and serotonin provide signal 
transmission in the retina as in the brain. Dopamine D2 receptors (D2R) are known to play an important role in the regulation of neural responses such as adaptation to light in the human retina (Masson 2019, Tian et al. 2015). There is insufficient information about the effects on the retina of psychotropic drugs used in schizophrenia and $\mathrm{BD}$ that affect the serotonergic and dopaminergic systems.

There are studies revealing that D2 and D3 receptors in the retina may decrease in drug-treated schizophrenia patients (Caravagio et al. 2018). Antipsychotics affect brain volume (Ho et al. 2011) and likewise modulate dopaminergic neurotransmission in the retina (Silverstein \& Rosen 2015). Electroretinogram (ERG) is a method in which retinal cell functions are electrophysiologically evaluated according to the paradigm (e.g., Flash ERG rod/cone functions, pattern ERG macular, bipolar, retina ganglion cell functions) and ERG studies showed abnormalities in schizophrenia and bipolar disorder (Jurišić et al. 2020). Findings in ERG studies are not specific in schizophrenia or bipolar disorder (Silić et al. 2020), however ERG might be utilized in psychopharmacology since the waves are determined by certain neurotransmitters. In a recently published metaanalysis, it was found that psychotropic drugs affect B waves in the electroretinogram as expected. (Youssef et al. 2019).

As the retina is dense in neurotransmitters, psychotropic drugs cause alterations in retinal physiology. The aim of this study was to examine the effects of psychotropic drugs used in the treatment of schizophrenia and $\mathrm{BD}$ on the RNFL and macular thickness.

\section{SUBJECTS AND METHODS}

\section{Study Design and Subjects}

This study was conducted in the psychiatry outpatient clinic of Trabzon Kanuni Training and Research Hospital between May 2017 and January 2018. According to DSM-5 diagnostic criteria 35 schizophrenia patients who had been using risperidone or clozapine for at least 6 months and 46 euthymic BD patients, aged 1865 years were included in the study. A control group was formed of 31 age and gender-matched healthy volunteers. Informed consent was obtained from all of the participants. The study protocol was convenient to Declaration of Helsinki and approved by Trabzon Kanuni Training and Research Hospital Ethics Committee (Date: June $07^{\text {th }}-2017$, Protocol\#: 2017/19).

The patients in the schizophrenia group were classified as those using risperidone or clozapine. The chlorpromazine equivalent risperidone doses (RD), chlorpromazine equivalent clozapine doses (CD) and chlorpromazine equivalent doses of all antipsychotics (APDs) were calculated (Langan et al. 2012). Schizophrenia patients using an additional antipsychotic to risperidone and clozapine at a dose exceeding the equivalent of $300 \mathrm{mg}$ chlorpromazine were not included in the study. In the BD group, the serum levels of valproate and lithium were measured and recorded. Chlorpromazine equivalent doses of antipsychotics taken in addition to the mood stabilizers were calculated (APDb).

Patients were excluded from the study if they were in a position to prevent giving consent to study participate, had mental retardation, any additional medical, psychiatric or neurological disease, any ocular pathology (glaucoma, a history of myopia $> \pm 2$ diopter or hypermetropia, ocular trauma, ocular surgery in the last 3 months), any condition such as hypertension or diabetes mellitus which could lead to macular degeneration or optic neuropathy, intracranial or intraocular lesions, a history of brain surgery, and in the control group, the presence of any axis 1 pathology according to the DSM-IV-TR diagnostic criteria, or the presence of any psychiatric disease in a first-degree relative.

\section{Assessments}

The Scale for the Assessment of Positive Symptoms (SAPS) (Andreasen 1984, Erkoc et al. 1991a) and the Scale for the Assessment of Negative Symptoms (SANS) (Andreasen 1983, Erkoc et al. 1991b) to determine severity of disease in the schizophrenia group, the Young Mania Rating Scale (YMRS) (Karadag et al. 2001, Young et al. 1978) and the Beck Depression Inventory (BDI) (Beck et al. 1961, Hisli 1988) to determine the severity of BD were applied.

For retinal evaluation, measurements of RNFL thickness and macular thickness were taken on an Optovue RTVue Premier (Optovue Inc., Fremont, California, USA) Optic Coherence Tomography device in the Ophthalmology Clinic of Kanuni Training and Research Hospital. RNFL imaging was applied using circular scans $3.4 \mathrm{~mm}$ in diameter in the center of the optic disc. RNFL thickness for the right (OD) and left (OS) eyes was automatically calculated in 7 regions of nasal $(\mathrm{N})$, superonasal (SN), inferonasal (IN), superotemporal (ST), temporal (T), and inferotemporal (IT), and global (G).

The macular thickness results were recorded from 9 regions of central foveal thickness $(\mathrm{CF})$, superior inner macula (SIM), superior outer macula (SOM), nasal inner macula (NIM), nasal outer macula (NOM), inferior inner macula (IIM), inferior outer macula (IOM), temporal inner macula (TIM), and temporal outer macula (TOM). Only well-centered, high quality images of $15 \mathrm{~dB}$ signal power were included in the study. During the course of the study, a total of 14 patients were excluded; 5 patients with refractive error $>2.00$ D, 1 with amblyopia, 2 determined with cataracts preventing OCT examination, and 6 diagnosed with HT or DM. 


\section{Statistical Analysis}

Sociodemographic characteristics were presented as descriptive statistics. Categorical variables were reported as number (n) and percentage (\%), and continuous variables as mean \pm standard deviation (SD) or median and interquartile range values. Distribution characteristics of the variables were assessed with the Shapiro Wilk test. Variables conforming to normal distribution were examined with parametric tests and those not showing normal distribution, with nonparametric tests. In the comparisons between the groups, the t-test or the Mann Whitney U-test was used. Categorical variables were compared using the Chi-square test. Analysis of variance (ANOVA) or the Kruskal Wallis test were applied to comparisons of 3 or more groups. For post-hoc comparisons, whether or not variances were homogenous was evaluated with the Levene's Test for Homogeneity of Variances. The Bonferroni Test was applied for homogenous variances and the Tamhane's Test for non-homogenous variances. To examine the relationships between variables, the Spearman Rank correlation test was applied. Linear Regression Analysis was performed to examine whether or not the measurements obtained on OCT were determined by other parameters. To examine whether or not the variables affected the differences between the groups, Univariate Covariance Analysis was applied. The results in all the tests were twotailed. A value of $p<0.05$ was accepted as statistically significant.

\section{RESULTS}

The sample consisted of 35 schizophrenia patients, $46 \mathrm{BD}$ patients and a control group of 31 healthy individuals. No statistically significant difference was determined between the groups in respect of age and gender. Also, no statistically significant difference was detected between the schizophrenia and BD groups in terms of chlorpromazine equivalent doses of antipsychotics, the duration of the last treatment received, or the number of hospitalizations. Age at onset is earlier and disease duration was significantly longer in the schizophrenia group (Table 1).

No statistically significant difference was determined between the schizophrenia, BD and healthy control groups in respect of macular thickness or RNFL in any area (Table 2).

In the correlation analyses of schizophrenia group, antipsychotic doses (APDs) were moderately positive corelated with RNLF regions of OD $\mathrm{T} \quad(\mathrm{r}=0.45$, $\mathrm{p}<0.01)$ and OS $\mathrm{T}(\mathrm{r}=0.58, \mathrm{p}<0.01)$, and moderately negative corelated with OS $\mathrm{N}(\mathrm{r}=-0.44, \mathrm{p}<0.01)$. Risperidone doses $(\mathrm{RD})$ were moderately negative correlated with OS $\mathrm{T}(\mathrm{r}=-0.62, \mathrm{p}<0.01)$, OS N $(\mathrm{r}=-0.52$, $\mathrm{p}<0.05)$, OS IN $(\mathrm{r}=-0.67, \mathrm{p}<0.01)$ area (Table 3$)$. In the $\mathrm{BD}$ group, moderately positive correlation was determined between the valproate dose and the macula regions of OD SOM $(\mathrm{r}=0.43, \mathrm{p}<0.05)$, OD SIM $(\mathrm{r}=0.38, \mathrm{p}<0.05)$, OD NOM $(\mathrm{r}=0.52, \mathrm{p}<0.01)$, OD TOM $(r=0.43, p<0.05)$, OD IOM $(r=-0.52, p<0.01)$,

Table 1. Sociodemographic data and psychiatric scale scores

\begin{tabular}{lcccc}
\hline & Schizophrenia $(\mathrm{n}=35)$ & Bipolar Disorder $(\mathrm{n}=46)$ & Healthy Control $(\mathrm{n}=31)$ & $\mathrm{p}$ \\
\hline Age* & $44.20 \pm 10.02$ & $42.17 \pm 13.39$ & $39.35 \pm 4.27$ & 0.170 \\
Gender n (\%) & & & & 0.530 \\
$\quad$ Female & $9(25 \%)$ & $17(36 \%)$ & $11(35 \%)$ & \\
$\quad$ Male & $26(75 \%)$ & $29(64 \%)$ & $20(64 \%)$ & 0.018 \\
Education n (\%) & & & $4(12.90 \%)$ & \\
$\quad$ Primary school & $18(51.42 \%)$ & $15(32.61 \%)$ & $19(61.29 \%)$ & \\
High school & $14(40.00 \%)$ & $24(52.17 \%)$ & $8(25.80 \%)$ & \\
$\quad$ University & $3(8.57 \%)$ & $7(15.22 \%)$ & & $<0.001$ \\
Smoker & $23(65.71 \%)$ & $29(63.04 \%)$ & $24(77.42 \%)$ & \\
Yes & $12(34.29 \%)$ & $17(36.96 \%)$ & $7(22.58 \%)$ & 0.027 \\
No & $22.91 \pm 8.02$ & $27.87 \pm 10.95$ & - & 0.001 \\
Age at onset* & $20.3 \pm 8.91$ & $13.26 \pm 8.69$ & - & 0.106 \\
Duration of disease* & $5.35 \pm 4.35$ & $4.02 \pm 2.93$ & - & $<0.001$ \\
Number of hospitalisations & $44.46 \pm 16.33$ & - & $0.23 \pm 0.71$ & $<0.001$ \\
SANS & $21.29 \pm 13.95$ & - & $0.19 \pm 0.60$ & $<0.001$ \\
SAPS & - & $8.54 \pm 7.90$ & $0.74 \pm 0.85$ & $<0.001$ \\
YMRS & & $2.63 \pm 2.24$ & $0.77 \pm 1.60$ & 0.100 \\
BDI & $283.43 \pm 141.93$ & $360.11 \pm 248.46$ & - & 0.120 \\
APD & $4.54 \pm 4.44$ & $2.47 \pm 2.69$ & - & \\
Duration of last treatment* & & & & \\
\hline
\end{tabular}

SAPS: Scale for the Assessment of Positive Symptoms; SANS: Scale for the Assessment of Negative Symptoms; YMRS: Young Mania Rating Scale; BDI: Beck Depression Inventory; APD: Antipsychotic dose; * Years 
Table 2. Comparison of retinal nerve fibre layer and macula thickness between controls and patients

\begin{tabular}{|c|c|c|c|c|c|}
\hline & Schizophrenia $(\mathrm{n}=35)$ & Bipolar Disorder $(\mathrm{n}=46)$ & Healthy Control $(n=31)$ & $\mathrm{F}$ & $\mathrm{p}$ \\
\hline OD SOM & $294.66 \pm 18.20$ & $303.04 \pm 35.34$ & $302.26 \pm 12.78$ & 1.18 & 0.300 \\
\hline OD SIM & $342.71 \pm 15.17$ & $352.37 \pm 59.82$ & $346.61 \pm 14.71$ & 0.59 & 0.554 \\
\hline OD NOM & $317.60 \pm 24.95$ & $321.28 \pm 21.25$ & $316.68 \pm 15.74$ & 0.59 & 0.592 \\
\hline OD NIM & $347.34 \pm 32.77$ & $339.07 \pm 34.39$ & $345.71 \pm 18.45$ & 0.85 & 0.427 \\
\hline OD TOM & $289.03 \pm 28.11$ & $288.02 \pm 20.09$ & $289.06 \pm 10.68$ & 0.03 & 0.969 \\
\hline OD TIM & $337.91 \pm 65.45$ & $324.93 \pm 31.78$ & $331.16 \pm 15.18$ & 0.92 & 0.400 \\
\hline OD IOM & $286.49 \pm 14.89$ & $300.30 \pm 51.70$ & $289.45 \pm 13.56$ & 1.77 & 0.175 \\
\hline OD IIM & $338.91 \pm 15.41$ & $338.50 \pm 31.00$ & $343.39 \pm 16.61$ & 0.45 & 0.634 \\
\hline OD CF & $285.31 \pm 87.30$ & $254.76 \pm 48.91$ & $262.23 \pm 17.14$ & 2.79 & 0.065 \\
\hline OS SOM & $296.63 \pm 17.30$ & $298.35 \pm 15.77$ & $347.94 \pm 12.40$ & 1.08 & 0.340 \\
\hline OS SIM & $339.40 \pm 16.48$ & $343.85 \pm 15.26$ & $347.94 \pm 12.40$ & 2.69 & 0.072 \\
\hline OS NOM & $315.91 \pm 18.17$ & $317.59 \pm 17.40$ & $318.00 \pm 14.20$ & 0.14 & 0.862 \\
\hline OS NIM & $341.46 \pm 16.18$ & $341.30 \pm 14.68$ & $342.35 \pm 24.34$ & 0.03 & 0.967 \\
\hline OS TOM & $281.54 \pm 14.18$ & $283.98 \pm 15.61$ & $286.71 \pm 10.83$ & 1.12 & 0.330 \\
\hline OS TIM & $327.34 \pm 15.08$ & $325.87 \pm 17.04$ & $331.23 \pm 14.21$ & 1.10 & 0.336 \\
\hline OS IOM & $287.46 \pm 16.06$ & $288.71 \pm 16.59$ & $289.48 \pm 13.65$ & 0.14 & 0.867 \\
\hline OS IIM & $336.11 \pm 17.83$ & $340.63 \pm 19.76$ & $342.77 \pm 15.80$ & 0.14 & 0.309 \\
\hline OS CF & $271.89 \pm 25.44$ & $262.89 \pm 22.01$ & $262.10 \pm 15.80$ & 2.22 & 0.113 \\
\hline OD ST (RNLF) & $135.71 \pm 37.51$ & $127.11 \pm 22.63$ & $134.90 \pm 18.15$ & 1.22 & 0.298 \\
\hline OD SN (RNLF) & $109.14 \pm 49.91$ & $101.29 \pm 18.33$ & $103.42 \pm 19.23$ & 0.61 & 0.544 \\
\hline OD T (RNLF) & $71.51 \pm 13.36$ & $70.09 \pm 10.17$ & $70.61 \pm 9.19$ & 0.16 & 0.848 \\
\hline OD N (RNLF) & $71.91 \pm 14.62$ & $75.24 \pm 26.44$ & $74.39 \pm 12.90$ & 0.28 & 0.753 \\
\hline OD IT (RNLF) & $138.66 \pm 17.57$ & $135.89 \pm 21.42$ & $142.00 \pm 20.03$ & 0.87 & 0.420 \\
\hline OD IN (RNLF) & $106.34 \pm 25.58$ & $104.67 \pm 30.67$ & $113.74 \pm 25.86$ & 1.04 & 0.357 \\
\hline OD G (RNLF) & $97.03 \pm 13.17$ & $94.76 \pm 15.30$ & $100.84 \pm 15.54$ & 1.57 & 0.212 \\
\hline OS ST (RNLF) & $132.49 \pm 14.09$ & $129.25 \pm 21.26$ & $135.06 \pm 18.93$ & 0.91 & 0.404 \\
\hline OS SN (RNLF) & $108.51 \pm 22.91$ & $105.32 \pm 19.90$ & $112.39 \pm 19.43$ & 1.05 & 0.352 \\
\hline OS T (RNLF) & $69.43 \pm 11.75$ & $71.20 \pm 12.47$ & $69.58 \pm 9.86$ & 0.29 & 0.749 \\
\hline OS N (RNLF) & $65.66 \pm 10.48$ & $66.22 \pm 18.57$ & $68.22 \pm 11.04$ & 0.29 & 0.746 \\
\hline OS IT (RNLF) & $140.66 \pm 21.69$ & $134.80 \pm 24.95$ & $143.26 \pm 20.52$ & 1.39 & 0.251 \\
\hline OS IN (RNLF) & $102.91 \pm 25.43$ & $101.16 \pm 22.57$ & $114.90 \pm 24.25$ & 3.32 & 0.040 \\
\hline OS G (RNLF) & $94.71 \pm 8.02$ & $93.29 \pm 13.65$ & $97.65 \pm 8.98$ & 1.48 & 0.232 \\
\hline
\end{tabular}

Note: 9 regions of macular thickness: CF: central foveal thickness; SOM: superior outer macula; SIM: superior inner macula; NOM: nasal outer macula; NIM: nasal inner macula; TOM: temporal outer macula; TIM: temporal inner macula; IOM: inferior outer macula; IIM: inferior inner macula;

7 regions of RNFL: ST: superotemporal; SN: superonasal; T: temporal; N: nasal; IT: inferotemporal; IN: inferonasal; G: global; OD: right eye; OS: left eye

Table 3. Schizophrenia correlation analyses

\begin{tabular}{lcccc}
\hline & APDs & RD & SANS & SAPS \\
\hline OD IIM & -0.06 & -0.33 & $-0.43^{* *}$ & -0.04 \\
OD T (RNFL) & $0.45^{* *}$ & 0.23 & -0.02 & 0.06 \\
OS T (RNFL) & $0.58^{* *}$ & $-0.62^{* *}$ & -0.16 & -0.26 \\
OS N (RNFL) & $-0.44^{* *}$ & $-0.52^{*}$ & 0.17 & 0.25 \\
OS IT (RNFL) & 0.32 & -0.01 & -0.12 & $-0.41^{*}$ \\
OS IN (RNFL) & -0.83 & $-0.67^{* *}$ & 0.04 & -0.26 \\
\hline
\end{tabular}

APDs: Antipsychotic dose of schizophrenia group; RD: Risperidon dose; OD: right eye; OS: left eye; IIM: inferior inner macula; T: Temporal; N: Nasal; IT: Inferotemporal; IN: inferonasal; * $\mathrm{p}_{0} 0.05 ; * * \mathrm{p}<0.01$ 
OS SOM $(r=0.36, p<0.05)$, OS NOM $(r=0.52, p<0.01)$ and $\mathrm{OS} \operatorname{IIM}(\mathrm{r}=0.36, \mathrm{p}<0.05)$ and a mild negative correlation was determined with OD TIM $(r=-0.36$, $\mathrm{p}<0.05$ ). A moderately positive correlation was determined between the valproate dose and the RNFL regions of OD G $(\mathrm{r}=0.50, \mathrm{p}<0.01)$, OS ST $(\mathrm{r}=0.39$, $\mathrm{p}<0.05)$, OS T $(\mathrm{r}=0.50, \mathrm{p}<0.01)$, OS N $(\mathrm{r}=0.42, \mathrm{p}<0.05)$, OS IT $(r=0.37, p<0.05)$, and OS G $(r=0.50, p<0.01)$ area. Serum valproate level was correlated positively at a moderate level with the macular region of OS NOM $(r=0.43, p<0.05)$ and with the RNLF regions of OS ST $(\mathrm{r}=0.41, \mathrm{p}<0.05)$ and OS T $(\mathrm{r}=0.57, \mathrm{p}<0.01)$. A negative correlation was determined between the antipsychotic doses and the OD CF $(r=-0.45, p<0.01)$ macular region and the RNFL regions of OS T $(\mathrm{r}=-0.31, \mathrm{p}<0.01)$ OS IT $(\mathrm{r}=-0.37, \mathrm{p}<0.01)$ and $\mathrm{OS} \mathrm{G}(\mathrm{r}=-0.33, \mathrm{p}<0.01)$ (Table 4).

Multiple linear regression analyses were applied to identify the determinative variables on RNFL and macular thickness in schizophrenia (Table 5) and BD (Table 6). In the schizophrenia group, in the left eye the APDs was determined to be a negative determinant of the OS SN region of RNLF $(\beta=-0.51 ; p<0.05)$, and the chlorpromazine equivalent dose of risperidone was detected to be a negative determinant of OS N $(\beta=-0.53$; $\mathrm{p}<0.05)$ and OS IN $(\beta=-0.81 ; \mathrm{p}<0.001)$ thickness (Table 5).

In the $\mathrm{BD}$ group, the serum valproate level was determined to be a positive determinant of the thickness of the macular regions of OD IOM $(\beta=0.46 ; p<0.05)$, OS NOM $(\beta=0.51 ; p<0.05)$, and OS IOM $(\beta=0.37$; $p<0.05)$, and the RNFL regions of OD IT $(\beta=0.52$; $p<0.05)$, OS T $(\beta=0.67 ; p<0.05)$, and OS IT $(\beta=0.40$; $\mathrm{p}<0.05$ ). The chlorpromazine equivalent dose of the whole antipsychotics (APD) was determined to be a negative predictor of right eye's CF thickness $(\beta=-0.71$; $\mathrm{p}<0.05$ ) (Table 6).

Table 4. Bipolar disorder correlation analyses

\begin{tabular}{llcccc}
\hline & Age & Age at onset & Valproate dose & Serum valproate level & APDb \\
\hline OD SOM & -0.27 & -0.21 & $0.43^{*}$ & 0.21 & -0.02 \\
OD SIM & -0.14 & -0.08 & $0.38^{*}$ & 0.16 & -0.01 \\
OD NOM & $-0.44^{* *}$ & -0.26 & $0.52^{* *}$ & 0.35 & -0.15 \\
OD TOM & $-0.40^{* *}$ & $-0.37^{*}$ & $0.43^{*}$ & 0.13 & -0.06 \\
OD TIM & -0.01 & 0.00 & $-0.36^{*}$ & 0.11 & -0.09 \\
OD IOM & -0.27 & -0.21 & $0.52^{* *}$ & 0.32 & -0.13 \\
OD CF & 0.23 & 0.17 & -0.20 & -0.04 & $-0.45^{* *}$ \\
OS SOM & $-0.38^{* *}$ & $-0.33^{*}$ & $0.36^{*}$ & 0.15 & -0.18 \\
OS NOM & $-0.46^{* *}$ & $-0.34^{*}$ & $0.52^{* *}$ & $0.43^{*}$ & -0.25 \\
OS TOM & $-0.48^{* *}$ & $-0.42^{* *}$ & 0.30 & 0.00 & -0.17 \\
OS IOM & $-0.52^{* *}$ & $-0.49^{* *}$ & 0.34 & 0.28 & -0.27 \\
OS IIM & -0.26 & -0.14 & $0.36^{*}$ & 0.17 & -0.11 \\
OD G (RNLF) & $-0.47^{* *}$ & $-0.39^{* *}$ & $0.50^{* *}$ & 0.36 & -0.25 \\
OS ST (RNLF) & $-0.40^{* *}$ & $-0.35^{*}$ & $0.39^{*}$ & $0.41 *$ & -0.20 \\
OS SN (RNLF) & -0.21 & $-0.33^{*}$ & 0.29 & 0.22 & -0.25 \\
OS T (RNLF) & -0.27 & -0.15 & $0.50^{* *}$ & $0.57^{* *}$ & $-0.31^{*}$ \\
OS N (RNLF) & -0.26 & -0.25 & $0.42^{*}$ & -0.05 & -0.11 \\
OS IT (RNLF) & $-0.39^{* *}$ & $-0.32^{*}$ & $0.37^{*}$ & 0.34 & $-0.37^{*}$ \\
OS IN (RNLF) & $-0.37^{*}$ & $-0.37^{*}$ & 0.13 & 0.16 & -0.22 \\
OS G (RNLF) & $-0.43^{* *}$ & $-0.40^{* *}$ & $0.50^{* *}$ & 0.35 & $-0.33^{*}$ \\
\hline
\end{tabular}

Note: Macular regions: SIM: superior inner macula; SOM: superior outer macula; CF: central foveal thickness,

NIM: nasal inner macula; NOM: nasal outer macula; IIM: inferior inner macula; IOM: inferior outer macula;

TIM: temporal inner macula; TOM: temporal outer macula;

RNFL regions: ST: superotemporal; SN: superonasal; T: temporal; N: nasal; IT: inferotemporal; IN: inferonasal; G: global; OD: right eye, OS:left eye; $* \mathrm{p}<0.05 ; * * \mathrm{p}<0.01$

Table 5. Schizophrenia regression analysis

\begin{tabular}{lcccccc}
\hline Predictive variable & Variable predicted & $\mathrm{B}$ & $\mathrm{t}$ & $\mathrm{R}$ & $\mathrm{R}^{2}$ & $\mathrm{~F}$ \\
\hline APDs & OS SN (RNLF) & -0.51 & -2.57 & 0.87 & 0.76 & $4.49^{*}$ \\
RD & OS N (RNLF) & -0.53 & -2.24 & 0.84 & 0.50 & $3.47^{*}$ \\
RD & OS IN (RNLF) & -0.81 & -3.25 & 0.73 & 0.54 & $2.97^{*}$ \\
\hline
\end{tabular}

APDs: antipsychotic dose of schizophrenia group; RD: Risperidon dose; N:nasal; SN: superonasal; IN: inferonasal; OS: left eye $* \mathrm{p}<0.05 ; \quad * * \mathrm{p}<0.01$ 
Table 6. Bipolar disorder regression analysis

\begin{tabular}{|c|c|c|c|c|c|c|c|}
\hline Group & Predictive variable & Variable predicted & B & $\mathrm{t}$ & $\mathrm{R}$ & $\mathrm{R}^{2}$ & $\mathrm{~F}$ \\
\hline \multirow{7}{*}{$\begin{array}{l}\overline{0} \\
\overline{0} \\
\dot{0} \\
\ddot{0} \\
\bar{\sigma} \\
\overline{0} \\
\stackrel{0}{0}\end{array}$} & \multirow{6}{*}{ Serum Valproate level } & OD IOM & 0.46 & 2.56 & 0.73 & 0.36 & $2.98^{*}$ \\
\hline & & OS NOM & 0.51 & 2.86 & 0.74 & 0.54 & $3.03^{*}$ \\
\hline & & OS IOM & 0.37 & 2.31 & 0.79 & 0.63 & $4.32 *$ \\
\hline & & OD IT (RNLF) & 0.52 & 2.73 & 0.68 & 0.47 & $2.27^{*}$ \\
\hline & & OS T (RNLF) & 0.67 & 4.08 & 0.78 & 0.61 & $4.05^{*}$ \\
\hline & & OS IT (RNLF) & 0.40 & 2.15 & 0.70 & 0.29 & $2.47^{*}$ \\
\hline & $\mathrm{APDb}$ & OD CF (RNLF) & -0.71 & -0.84 & 0.74 & 0.55 & $3.19^{*}$ \\
\hline
\end{tabular}

APDb: antipsychotic dose of bipolar disorder group; OD: right eye; OS: left eye; Macular sub-regions: NOM: nasal outer macula, IOM: inferior outer macula, RNLF sub-regions: T: Temporal, IT: Inferotemporal, CF: central foveal thickness

$* \mathrm{p}<0.05 ; \quad * * \mathrm{p}<0.01$

\section{DISCUSSION}

Most important findings of this study were that the serum valproate level was a positive predictor for the thickness of right inferior outer macula, the left inferior outer macula and left nasal outer macula, RNFL subregions of right, left inferotemporal and left temporal regions in BD. Another important finding was that antipsychotic chlorpromazine equivalent doses were a negative predictor of thickness in certain optic nerve regions in schizophrenia and BD. In schizophrenia patients, the chlorpromazine equivalent risperidone doses were determined to be a negative predictor of left nasal and left inferonasal region thickness. No significant difference was determined between the BD and schizophrenia groups in the current study in macular thickness.

In the BD patients, the serum valproate level was a positive predictor of the thickness of the macula and RNFL sub-regions. However, the relationship between these effects and the clinical effects could not be fully understood in this study. Clinical parameters such as age, gender, metabolic causes, number of episodes, age of onset of disease were evaluated, but there was no relationship between response to treatment or other clinical measures and the effects of valproate on the retina. In a study with epilepsy patients, there was a relationship between valproate and a reduction in retinal thickness, especially in the superior and inferior quadrants (Xiong et al. 2019). These findings were not consistent with current study, but it was reported in a previous study that epilepsy itself could have been another risk factor for the reduction in retinal thickness. There is evidence that valproate has neuroprotective effects on the optic nerve and macula, and recent studies have shown some promising results in neurodegenerative disease models such as glaucoma, Alzheimer's disease and Parkinson's disease (Monti et al. 2010, Qing et al. 2008).

As a gamma aminobutyric acid (GABA) transaminase inhibitor, sodium channel blocker and histone deacetylase (HDAC) inhibitor, valproate have been used for almost 50 years in the treatment of epilepsy, mood disorders, migraine and neuropathic pain (Gottlicher et al. 2001). Valproate has been reported to stimulate the BDNF-TrkB pathway and to be neuroprotective by reducing glutamate excitotoxicity (Friedmann et al. 2004, Kimura et al. 2015). It also prevents apoptosis in the retina by reducing ER stress by regulating gene expression through epigenetic mechanisms (Zhang et al. 2011). With these effects, valproate produces trophic effects in neural tissues, and therefore the findings in our study are the results of these effects in the retina.

Another finding of the current study was that antipsychotic chlorpromazine equivalent doses were a negative predictor of thickness in certain optic nerve regions in schizophrenia and BD. In the schizophrenia group, the chlorpromazine equivalent doses of risperidone were determined to be negative predictors of OS N and OS IN thickness. No such effect was determined for clozapine. These findings suggest that these changes in the retina are the result of dopaminergic blockage, since the major difference between risperidone and clozapine is risperidone blocks dopaminergic receptors much higher than clozapine. One result of DA receptor blockage in the retina is less input to the retinal ganglion cells. In addition, it has been reported to cause amplitude loss in the B-waves of dopamine agonists on electroretinogram, and vice versa, that dopaminergic drugs lead to amplitude increase in the B-waves (Youssef et al. 2019). This has been suggested as the mechanism explaining ganglion cell atrophy in Parkinson's disease (Tian et al. 2011). As antipsychotic chlorpromazine equivalent doses were a negative predictor of thickness in certain optic nerve regions in schizophrenia and BD in the current study, this suggests that dopaminergic blockage could cause ganglion cell atrophy. There are studies supporting this claim, which have shown that the use of antipsychotics causes a decrease in the volume of grey matter in the brain (Cahn et al. 2002, Lieberman et al. 2005, Zhuo et al. 2020).

Small sample size of the study limits the generalizability of the results. Various factors that may have affected the macular and RNLF thickness such as the multiple antipsychotic use of patients and smoking in the patient and control groups are among the most important limitations of this study. There is a need for further studies with larger patient groups and longer follow-up to confirm these results. However, consistency of the findings with the literature is the strength of the study. 


\section{CONCLUSION}

The results that valproate has neuroprotective effects on the optic nerve and macula were consistent with previous evidence. Examination of the retina may be useful for the evaluation of the effects of psychopharmalogical treatments. Today individualized treatments are on the agenda, and monitoring of the amount of mood-regulating drugs in the body with the of serum drug level monitoring, but we have very little information to be able to evaluate the response to treatments in BD (Joshi et al. 2019). Based on the findings obtained in these studies, biological markers may be helpful in the evaluation of response to treatment of patients.

\section{Acknowledgements:}

This research did not receive any specific grant from funding agencies in the public, commercial, or not-forprofit sectors.

\section{Conflict of interest: None to declare.}

\section{Contribution of individual authors:}

Ilkay Keles Altun \& Murat Ilhan Atagun have performed literature review, developed the hypothesis and study protocol and obtained ethical committee approval and prepared the manuscript and submitted the manuscript

Ilkay Keles Altun, Neslihan Turedi \& Neriman Aras enrolled the patients and obtained the data.

Murat Ilhan Atagun has performed data analysis.

\section{References}

1. Andreasen NC: The scale for the assesment of negative symtoms (SANS). University of Iowa Press, Iowacity, 1983

2. Andreasen NC: The scale for the assesment of positive symtoms (SAPS), University of Iowa Press, Iowacity, 1984

3. Appaji A, Nagendra B, Chako DM, Padmanabha A, Jacob $A$, Hiremath $C V$ et al: Examination of retinal vascular trajectory in schizophrenia and bipolar disorder. Psychiatry Clin Neurosci 2019; 73:738-44. doi:10.1111/pcn.12921

4. Ascaso FJ, Rodriguez-Jimenez R, Cabezón L, López-Antón $R$, Santabárbara J, De la Cámara $C$ et al: Retinal nerve fiber layer and macular thickness in patients with schizophrenia: Influence of recent illness episodes. Psychiatry Res 2015; 229:230-36 Doi: 10.1016/j.psychres.2015.07.028

5. Beck AT, Ward CH, Mendelson M, Mock J, Erbaugh J: An inventory for measuring depression. Arch Gen Psychiatry 1961; 4:561-71 doi:10.1001/archpsyc.1961.01710120031004

6. Buoli M, Serati M, Caldiroli A, Cremaschi L, Altamura AC: Neurodevelopmental Versus Neurodegenerative Model of Schizophrenia and Bipolar Disorder: Comparison with Physiological Brain Development and Aging. Psychiatr Danub 2017; 29:24-7. doi:10.24869/psyd.2017.24

7. Cahn W, Hulshoff Pol HE, Lems EB, van Haren NE, Schnack HG, van der Linden JA et al: 2002. Brain volume changes in first-episode schizophrenia: a 1-year follow-up study. Arch Gen Psychiatry 2002; 59:1002-10. doi:10.1001/archpsyc.59.11.1002
8. Caravaggio F, Scifo E, Sibille EL, Hernandez-Da Mota SE, Gerretsen P, Remington G, Graff-Guerrero A: 2018. Expression of dopamine D2 and D3 receptors in the human retina revealed by positron emission tomography and targeted mass spectrometry. Exp Eye Res 2018; 175:32-41. doi:10.1016/j.exer.2018.06.006

9. Chu EM, Kolappan M, Barnes TR, Joyce EM, Ron MA: A window into the brain: an in vivo study of the retina in schizophrenia using optical coherence tomography. Psychiatry Res 2012; 203:89-94. doi:10.1016/j.pscychresns.2011.08.011

10. Erkoc,Ş, Arkonac, O, Atakl C, Özmen E: The reliability and validity of scale for the assesment of the positive symptoms. Dusunen Adam: The Journal of Psychiatry and Neurological Sciences 1991a; 4:20-4

11. Erkoc, Ş, Arkonac, O, Atakl C, Özmen E: 1991b. The reliability and validity of scale for the assesment of the negative symptoms. Dusunen Adam: The Journal of Psychiatry and Neurological Sciences 1991b; 4:16-9

12. Friedman SD, Dager SR, Parow A, Hirashima F, Demopulos $C$, Stoll $A L$ et al: Lithium and valproic acid treatment effects on brain chemistry in bipolar disorder. Biol Psychiatry 2004; 56:340-48. doi:10.1016/j.biopsych.2004.06.012

13. Gigante AD, Young LT, Yatham LN, Andreazza AC, Nery $F G$, Grinberg LT et al: Morphometric post-mortem studies in bipolar disorder: possible association with oxidative stress and apoptosis. Int J Neuropsychopharmacol 2011; 14:1075-89. doi:10.1017/S146114571000146X

14. Göttlicher M, Minucci S, Zhu P, Krämer OH, Schimpf A, Giavara $S$, et al: Valproic acid defines a novel class of $H D A C$ inhibitors inducing differentiation of transformed cells. The EMBO J 2001; 20:6969-78. https://doi.org /10.1093/emboj/20.24.6969

15. Hisli N: Beck Depresyon Envanterinin gecerligĭ uz̈erine bir callsma. Psikoloji Dergisi 1988; 6:118-22

16. Ho BC, Andreasen NC, Ziebell S, Pierson R, Magnotta V: Long-term antipsychotic treatment and brain volumes: a longitudinal study of first-episode schizophrenia. Arch Gen Psychiatry 2011; 68:128-137.

doi:10.1001/archgenpsychiatry.2010.199

17. Honea R, Crow TJ, Passingham D, Mackay CE: Regional deficits in brain volume in schizophrenia: a meta-analysis of voxel-based morphometry studies. Am J Psychiatry 2005; 162:2233-45. doi:10.1176/appi.ajp.162.12.2233

18. Joshi A, Bow A, Agius M: Pharmacological Therapies in Bipolar Disorder: a Review of Current Treatment Options. Psychiatr Danub 2019; 31(Suppl 3):595-603

19. Jurišić D, Ćavar I, Sesar A, Sesar I, Vukojević J, Ćurković M: New Insights into Schizophrenia: a Look at the Eye and Related Structures Psychiatr Danub 2020; 32:60-9. doi:10.24869/psyd.2020.60

20. Karadag F, Oral ET, Yalcin F, Erten E: Reliability and validity of Turkish translation of Young Mania Rating Scale. Turkish Journal of Psychiatry. 2002; 13:107-14

21. Kempton MJ, Geddes JR, Ettinger U, Wiliams SCR, Grasby PM: Meta-analysis, database, and meta-regression of 98 structural imaging studies in bipolar disorder. Arch Gen Psychiatry 2008; 65:1017-32. doi:10.1001/archpsyc.65.9.1017

22. Kimura A, Namekata K, Guo X, Noro T, Harada C, Harada T: Valproic acid prevents NMDA-induced retinal ganglion cell death via stimulation of neuronal TrkB receptor signaling. Am J Pathol 2015; 185:756-64. doi:10.1016/j.ajpath.2014.11.005 
23. Langan J, Martin D, Shajahan P, Smith DJ: Antipsychotic dose escalation as a trigger for neuroleptic malignant syndrome (NMS): literature review and case series report. BMC Psychiatry 2012; 12:214. doi:10.1186/1471-244X-12-214

24. Lieberman JA, Tollefson GD, Charles C, Zipursky $R$, Sharma T, Kahn RS et al: Antipsychotic drug effects on brain morphology in first-episode psychosis. Arch Gen Psychiatry 2005; 62:361-70 doi:10.1001/archpsyc.62.4.361

25. Lizano P, Bannai D, Lutz O, Kim LA, Miller J, Keshavan M: A Meta-analysis of Retinal Cytoarchitectural Abnormalities in Schizophrenia and Bipolar Disorder. Schizophr Bull 2020; 46:43-53. doi:10.1093/schbul/sbz029

26. Lu Y, Li Z, Zhang X, Ming B, Jia J, Wang R, Ma D: Retinal nerve fiber layer structure abnormalities in early Alzheimer's disease: evidence in optical coherence tomography. Neurosci Lett 2010; 480:69-72. doi:10.1016/j.neulet.2010.06.006

27. Martin EG, Gavin A, Campayo JG, Vilades E, Orduna E, Polo V et al: Visual function and retinal changes in patients with bipolar disorder. Retina 2019; 39:2012-21

28. Masson J: Serotonin in retina. Biochimie 2019;161:51-5 Doi: 10.1016/j.biochi.2018.11.006

29. Monti B, Gatta V, Piretti F, Raffaelli SS, Virgili M, Contestabile A: Valproic acid is neuroprotective in the rotenone rat model of Parkinson's disease: involvement of alpha-synuclein. Neurotox. Res. 2010; 17:130-41 Doi: 10.1007/s12640-009-9090-5

30. Moorhead TW, McKirdy J, Sussmann JE, Hall J, Lawrie SM, Johnstone EC, McIntosh AM: Progressive gray matter loss in patients with bipolar disorder. Biol Psychiatry 2007; 62:894-900 Doi: 10.1016/j.biopsych.2007.03.005

31. Moschos MM, Tagaris G, Markopoulos I, Margetis I, Tsapakis S, Kanakis M, Koutsandrea C: Morphologic changes and functional retinal impairment in patients with Parkinson disease without visual loss. Eur J Ophthalmol 2011;21:24-9 Doi: 10.5301/ejo.2010.1318

32. Narayanan B, O'Neil K, Berwise C, Stevens MC, Calhoun $V D$, Clementz BA et al: Resting state electroencephalogram oscillatory abnormalities in schizophrenia and psychotic bipolar patients and their relatives from the bipolar and schizophrenia network on intermediate phenotypes study. Biol Psychiatry 2014; 76:456-65. doi:10.1016/j.biopsych.2013.12.008

33. Nudmamud S, Reynolds LM, Reynolds GP: N-acetylaspartate and $N$-Acetylaspartylglutamate deficits in superior temporal cortex in schizophrenia and bipolar disorder: a postmortem study. Biol Psychiatry 2003; 53:1138-41. doi:10.1016/s0006-3223(02)01742-0

34. Pan J, Zhou Y, Xiang Y, Yu J: Retinal nerve fiber layer thickness changes in Schizophrenia: A meta-analysis of case-control studies. Psychiatry Res 2018; 270:786-91. doi:10.1016/j.psychres.2018.10.075

35. Qing H, He G, Ly PT, Fox CJ, Staufenbiel M, Cai F et al: Valproic acid inhibits Abeta production, neuritic plaque formation, and behavioral deficits in Alzheimer's disease mouse models. J Exp Med 2008; 205:2781-89. doi:10.1084/jem.20081588
36. Silić A, Ostojić D, Karlović D: ERG and OCT as an Effective Screening and Staging Tools for Schizophrenia? Psychiatr Danub 2020; 32:74-75

37. Silverstein SM, Rosen R: Schizophrenia and the eye. Schizophrenia Res Cogn 2015; 2:46-55. doi:10.1016/j.scog.2015.03.004

38. Thiele S, Nadal J, Fleckenstein M, Fang PP, Pfau M, Schmid M et al: Longitudinal Analysis of Drusen Volume in Intermediate Age-Related Macular Degeneration Using Two Spectral-Domain Optical Coherence Tomography Scan Patterns. Ophthalmologica 2018; 239:110-20. doi:10.1159/000485260

39. Tian N, Xu HP, Wang P: Dopamine D2 receptors preferentially regulate the development of light responses of the inner retina. Eur J Neurosci 2015; 41:17-30 Doi: 10.1111/ejn.12783

40. Tian T, Zhu XH, Liu YH: Potential role of retina as a biomarker for progression of Parkinson's disease. Int $J$ Ophthalmol 2011; 4:433-38. doi:10.3980/j.issn.2222-3959.2011.04.21

41. Topcu-Yilmaz P, Aydin M, Cetin Ilhan B: Evaluation of retinal nerve fiber layer, macular, and choroidal thickness in schizophrenia: spectral optic coherence tomography findings. Psychiatry and Clinical Psychopharmacology 2019; 29:28-33 Doi: 10.1080/24750573.2018.1426693

42. Whiteford HA, Ferrari AJ, Degenhardt L, Feigin V, Vos T: 2015. The global burden of mental, neurological and substance use disorders: an analysis from the Global Burden of Disease Study 2010. PLoS One 2015; 10:e0116820. doi:10.1371/journal.pone.0116820

43. Xiong W, Lu L, Zhang Y, Xiao Y, Gao H, Zhang M, Zhou D: Attenuation of retinal nerve fibre layer in people with epilepsy receiving valproate. Epilepsy Res 2019; 154:1448. Doi: 10.1016/j.eplepsyres.2019.05.015

44. Young RC, Biggs JT, Ziegler VE, Meyer DA: A rating scale for mania: reliability, validity and sensitivity. $\mathrm{Br} J$ Psychiatry 1978; 133:429-35. Doi: 10.1192/bjp.133.5.429

45. Youssef P, Nath S, Chaimowitz GA, Prat SS: Electroretinography in psychiatry: A systematic literature review. Eur Psychiatry 2019; 62:97-106. doi:10.1016/j.eurpsy.2019.09.006

46. Yusifova A, Atagün MI, Uğurlu N, Malekghasemi S, Şenat $A$, Demirlek C, Erel Ö: Retinal fundus imaging in bipolar disorder: A pilot study. Psychiatry Clin Neurosci 2020 ; 74:85-6 doi: 10.1111/pcn.12951

47. Zhang Z, Tong N, Gong Y, Qiu Q, Yin L, Lv X, Wu X: Valproate protects the retina from endoplasmic reticulum stress-induced apoptosis after ischemia-reperfusion injury. Neurosci Lett 2011; 504:88-92. doi:10.1016/j.neulet.2011.09.003

48. Zhuo C, Xiao B, Chen C, Jiang D, Li G, Ma X et al: Antipsychotic agents deteriorate brain and retinal function in schizophrenia patients with combined auditory and visual hallucinations: A pilot study and secondary followup study. Brain and Behav 2020; 10:e01611. doi:10.1002/brb3.1611

\section{Correspondence:}

Murat Ilhan Atagun MD, PhD

Department of Psychiatry, Izmir Bakurcay University Faculty of Medicine

Gazi Mustafa Kemal Region, Kaynaklar Street, 35665 Menemen/Izmir, Turkey

E-mail: ilhan.atagun@gmail.com 